\title{
Vacuolar protein sorting 35 (Vps35) rescues locomotor deficits and shortened lifespan in Drosophila expressing a Parkinson's disease mutant of Leucine-rich repeat kinase 2 (LRRK2)
}

\author{
Radek Linhart ${ }^{\dagger}$, Sarah Anne Wong ${ }^{\dagger}$, Jieyun Cao ${ }^{\dagger}$, Melody Tran, Anne Huynh, Casey Ardrey, Jong Min Park,
} Christine Hsu, Saher Taha, Rentia Peterson, Shannon Shea, Jason Kurian and Katerina Venderova*

\begin{abstract}
Background: Parkinson's disease (PD) is the most common movement neurodegenerative movement disorder. An incomplete understanding of the molecular pathways involved in its pathogenesis impedes the development of effective disease-modifying treatments. To address this gap, we have previously generated a Drosophila model of PD that overexpresses PD pathogenic mutant form of the second most common causative gene of PD, Leucine-Rich Repeat Kinase 2 (LRRK2).

Findings: We employed this model in a genetic modifier screen and identified a gene that encodes for a core subunit of retromer - a complex essential for the sorting and recycling of specific cargo proteins from endosomes to the trans-Golgi network and cell surface. We present evidence that overexpression of the Vps35 or Vps26 component of the cargo-recognition subunit of the retromer complex ameliorates the pathogenic mutant LRRK2 eye phenotype. Furthermore, overexpression of Vps35 or Vps26 significantly protects from the locomotor deficits observed in mutant LRRK2 flies, as assessed by the negative geotaxis assay, and rescues their shortened lifespan. Strikingly, overexpressing Vps35 alone protects from toxicity of rotenone, a neurotoxin commonly used to model parkinsonism, both in terms of lifespan and locomotor activity of the flies, and this protection is sustained and even augmented in the presence of mutant LRRK2. Finally, we demonstrate that knocking down expression of Vps35 in dopaminergic neurons causes a significant locomotor impairment.
\end{abstract}

Conclusions: From these results we conclude that LRRK2 plays a role in the retromer pathway and that this pathway is involved in PD pathogenesis.

Keywords: Parkinson's disease, LRRK2, VPS35, Retromer, Endolysosomal pathway, Drosophila, Genetics, Rotenone, Neurodegeneration, Endosomes, Lysosome, VPS26

\section{Background}

A growing unmet need for better treatments of neurodegenerative disorders, including Parkinson's disease (PD), highlights the importance of research into the pathological mechanisms involved in the disease process.

Identification of several causative genes has led to new insights into PD pathogenesis. Leucine-Rich Repeat Kinase

\footnotetext{
* Correspondence: kvenderova@pacific.edu

${ }^{\dagger}$ Equal contributors

Department of Physiology and Pharmacology, Thomas J. Long School of Pharmacy and Health Sciences, University of the Pacific, 751 Brookside Rd,
} Stockton, CA 95211, USA
2 (LRRK2) (GenBank: AY792511) is the second most common causative gene of PD. Thus far, seven point mutations within LRRK2 have been demonstrated to segregate with the disease and numerous common and rare LRRK2 gene variants that increase susceptibility to PD have been described. LRRK2 has also been linked to tau [1] and $\alpha$-synuclein [2-4] pathologies and therefore may be a key player upstream of cell death pathways involved in other neurodegenerative processes [5]. LRRK2 is a kinase with a Roc-COR catalytic core that has a sequence homology to Rab GTPases. Other domains include LRR and 
WD-40, predicted to be involved in protein-protein interactions. Despite promising new findings, exactly how LRRK2 contributes to cell death/survival and what is its physiological function, still remains largely unknown.

Although no animal model developed thus far has been able to reproduce all key pathological features of PD, transgenic Drosophila models have proven particularly useful, as they faithfully reproduce dopaminergic (DA) neuronal death and locomotor deficits [6-8]. Drosophila melanogaster is a highly suitable model organism for studies of gene function, interactions and elucidation of genetic pathways. Notably, Drosophila compound eye can be successfully employed in unbiased genome-wide genetic modifier screens in vivo [9-11]. Results from such screens and other research in Drosophila have recently generated important new insights into the pathophysiology of several neurodegenerative disorders, including PD [12].

To help dissect the molecular processes involved in PD pathology, we recently generated a Drosophila overexpressing human LRRK2 with a PD pathogenic I2020T mutation within the kinase domain [13]. This transgenic model has been successfully used by other researchers $[14,15]$. As shown previously by our team [13] and independently validated by others [16-18], expressing pathogenic mutant LRRK2 in Drosophila DA neurons recapitulates many of the cardinal features of $\mathrm{PD}$, including the loss of DA neurons and locomotor deficits [13]. In addition, LRRK2 mutant flies present with an abnormal eye phenotype, allowing us to perform an in vivo genetic modifier screen in search for genetic interactors of $L R R K 2$. Here, we provide evidence that LRRK2 genetically interacts with Vacuolar protein sorting 35 (Vps35) (GenBank: AE013599.4), a core component of the retromer complex.

\section{Results \\ Vps35 partially rescues the eye phenotype of flies expressing pathogenic mutant LRRK2}

In our studies, we employed the commonly used UAS-Gal4 system for a cell/tissue-specific expression [19]. As we have previously shown [13], expression of one of the PD-causing mutants of LRRK2, LRRK2(I2020T), under an eye-specific $(G M R)$ promoter at $29^{\circ} \mathrm{C}$ causes a rough eye phenotype with pigmentation deficits. Notably, 50.03\%+/-6.58\% of LRRK2(I2020T) eyes have black lesions (Figure 1). Similar lesions were reported in other fly models of neurodegeneration [20-24] and seem to be indicative of neuronal (photoreceptor) death occurring later in the eye development, after a full differentiation [21]. Such black lesions are very rare in control (GMR alone) flies $(3.03 \%+/-3.03 \%$ of eyes) (Figure 1). Employing this LRRK2(I2020T) eye phenotype as a read-out in a genetic modifier screen, we identified Vacuolar protein sorting 26 (Vps26) (GenBank:
NM_130596.2) as a new LRRK2 interacting gene. Specifically, overexpressing endogenous Drosophila Vps26 in the eye caused a mild eye phenotype, including an occasional presence of black lesions (11.21\% +/- 2.12\% of flies) (Figure 1A and B). Strikingly, overexpressing Vps26 in the $L R R K 2(I 2020 T)$ flies rescued the black lesion eye phenotype of the LRRK2 mutants $(10.10 \%+/-2.12 \%$; $\mathrm{P}<0.05 ; \mathrm{F}(3,9)=13.30$ ) (Figure $1 \mathrm{~A}$ and $\mathrm{B}$ ).

Vps26, together with Vps35 and Vps29, are the three core components of the cargo-recognition subunit of the retromer complex. Because human homologue of Vps35, VPS35 (GenBank: NC_000016.9), has been recently identified as a new candidate PD gene $(3,4)$, our first goal was to determine whether $L R R K 2$ also genetically interacts with Vps35.

Drosophila Vps35 (CG5625) is located on the right arm of the second chromosome and has $61 \%$ identity with its human homologue. Again, an eye-specific overexpression of endogenous Vps35 alone caused a mild eye phenotype that included the occasional presence of black lesions (7.56\% of eyes, +/- 3.92\%) (Figure 1A and C). Similar to Vps26, increased Vps35 expression completely rescued the black lesion phenotype of LRRK2 mutants: none of the analyzed eyes displayed any black lesions $(\mathrm{P}<0.0001 ; \mathrm{F}$ $(2,6)=102.3)$ (Figure $1 \mathrm{~A}$ and $\mathrm{C}$ ). This suggests that both Vps26 and Vps35 genetically interact with $L R R K 2$ in the Drosophila eye.

\section{Vps35 rescues the locomotor and lifespan deficits of flies expressing pathogenic mutant LRRK2}

One of the cardinal characteristics of PD is a loss of DA neurons which has a negative impact on movement. Therefore, in our next experiments we used a Dopa-decarboxylase-Gal4 (Ddc-Gal4) driver line that is commonly used in Drosophila PD research to target gene expression to DA neurons [6,25].

To quantify locomotor activity, we employed a wellestablished negative geotaxis climbing assay. As we have shown previously, overexpressing LRRK2(I2020T) in DA neurons causes significant locomotor deficits [13]. Compared to control, the climbing ability of LRRK2(I2020T) flies was reduced by $61.45 \%+/-7.49 \%$ on day 5 (specifically, $28.04 \%+/-5.45 \%$ of $L R R K 2$ flies were able to reach the line within 5 seconds, compared to $72.75 \%+/-7.89 \%$ of control) (Figure 2). Similar to the eye, this LRRK2 phenotype can be fully rescued either by overexpressing $V p s 35$ in DA neurons (70.00\% +/- 6.36\% of flies overexpressing Vps35 and mutant LRRK2 were able to cross the line within 5 seconds) $(\mathrm{P}<0.0001 ; \mathrm{F}(4,77)=8.20)$ (Figure $2 \mathrm{~B})$, or by overexpressing Vps26 (64.12\% +/- 3.28\% of flies overexpressing Vps 26 and mutant $L R R K 2$ in DA neurons crossed the line within 5 seconds) $(\mathrm{P}<0.0001$; F $(3,225)=11.31)$ (Figure $2 \mathrm{~A})$. Similar to day 5, Vps35 or Vps26 overexpression rescued the LRRK2(I2020T) phenotype on day 10 (Figure 2A and B). By 


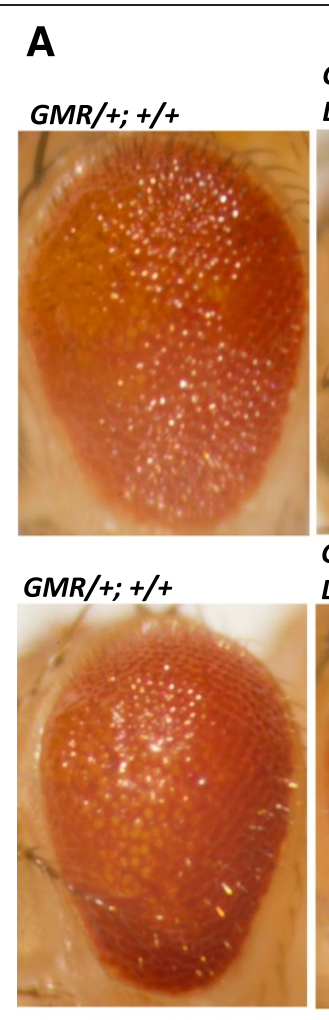

B

\section{GMR/+;}

LRRK2(I2020T)/+

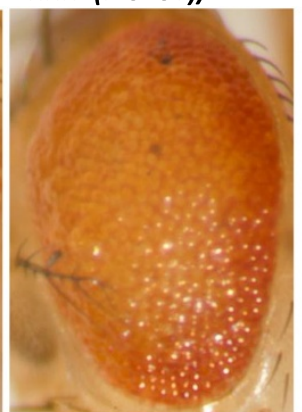

GMR/+; LRRK2(I2020T)/+
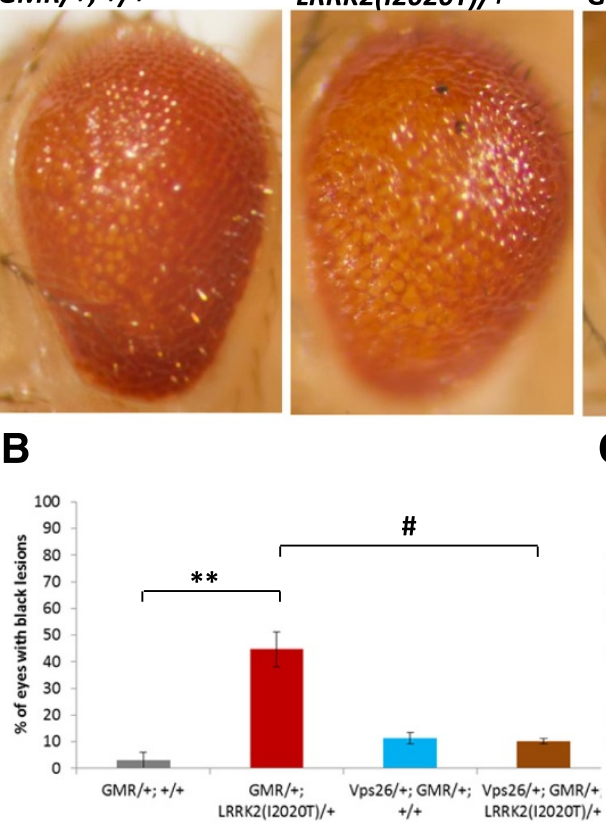

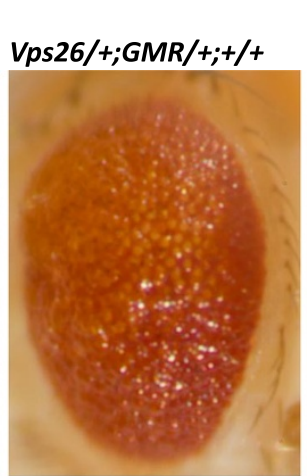

Vps26/+;GMR/+;

LRRK2(I2020T)/+

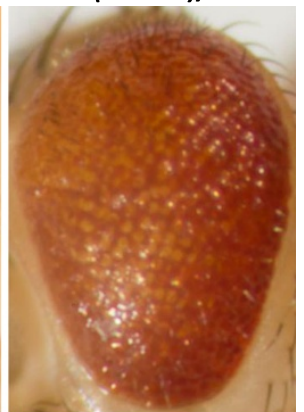

GMR/Vps35;

GMR/Vps35; +/+

LRRK2(I2020T)/+
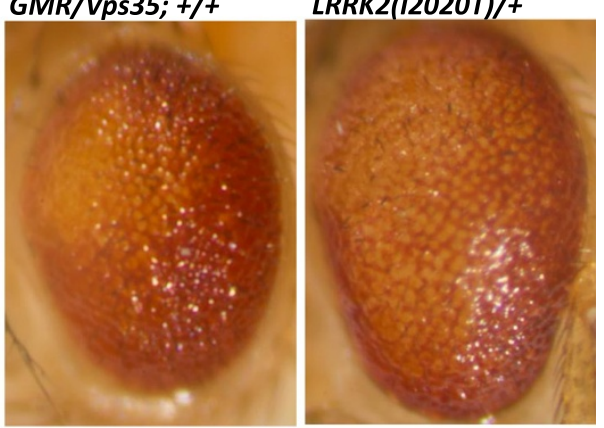

C

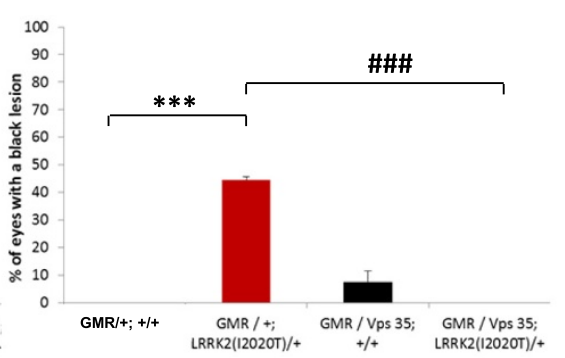

Figure 1 Eye-specific overexpression of Vps26 or Vps35 rescues the black lesion phenotype caused by expression of LRRK2(I2020T). A) Representative stereomicroscope images of eyes overexpressing Vps26 (upper panel) and Vps35 (lower panel). B) Quantification of black lesions in flies overexpressing Vps26. Total of 314 eyes from 2-6 independent crosses/genotype (males and females) was analyzed. C) Quantification of black lesions in flies overexpressing Vps35. Total of 312 eyes from 3 independent crosses/genotype (males and females) was analyzed. All flies were reared at $29^{\circ} \mathrm{C}$. Statistical analysis by One-Way ANOVA, Bonferroni's post-test ( $\left.\mathrm{P}<0.0001\right)$. Statistically significant difference compared to control (GMR alone) is denoted as ** for $\mathrm{P}<0.001$, or *** for $\mathrm{P}<0.0001$. Statistically significant difference compared to GMR/+;LRRK2(I2020T)/+is denoted as \# for $\mathrm{P}<0.05$, or \#\#\# for $\mathrm{P}<0.0001$.

day 20 however, the locomotor activity of all flies, including control, was severely impaired due to age (Figure 2A and B). Please note that the locomotor activity of flies was always assessed at three different time intervals $(5,10$ and 30 seconds), with similar results (data not shown). In addition to the locomotor activity, we also assessed survival of these flies. Compared to the control ( $D d c$ alone), survival of flies expressing LRRK2(I2020T) in DA neurons was significantly shorter. This phenotype was completely rescued by overexpression of Vps35 (Figure 3).
Altogether, these data validate that mutant LRRK2(I2020T) functionally interacts with Vps35 and Vps26 in DA neurons and that this interaction is important for the locomotor activity and basal survival of the flies.

\section{Overexpression of $V p s 35$ rescues locomotor deficits of other LRRK2 mutants}

The I2020T substitution is localized within the kinase domain of $L R R K 2$. Our next question was to determine whether the functional interaction between $L R R K 2$ and 

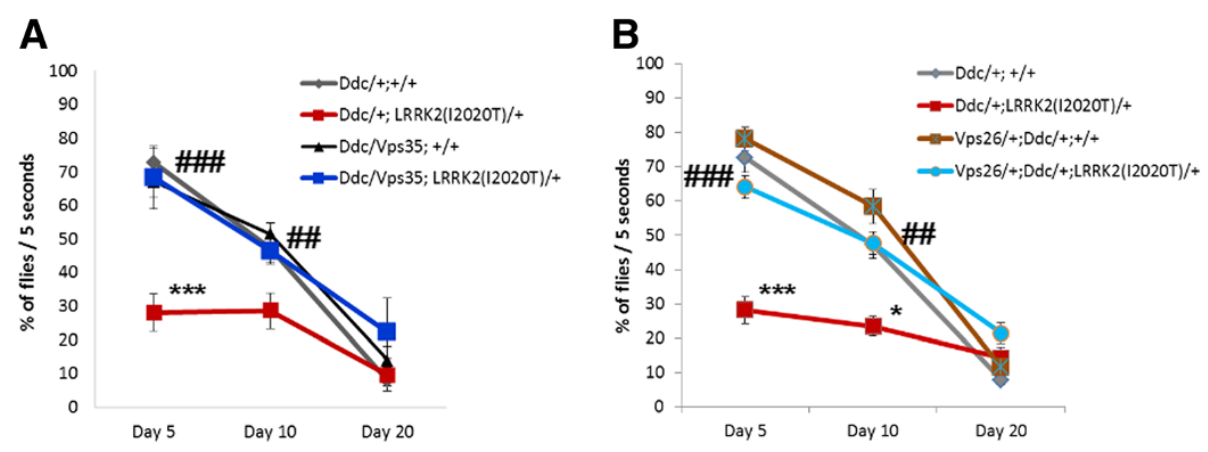

Figure 2 Overexpression of Vps26 or Vps35 in DA neurons rescues LRRK2(I2020T) locomotor deficits. (A) Overexpression of Vps26 in DA neurons. (B) Overexpression of Vps35 in DA neurons. $\mathrm{N}=3-12$ cohorts of ten per genotype. All flies were reared at $29^{\circ} \mathrm{C}$. Statistical analysis: Two-Way ANOVA, Tukey's post-test. Statistically significant difference compared to control (Ddc alone) is denoted as * for $P<0.05$ and *** for $P<0.0001$. Statistically significant difference compared to Ddc/t,LRRK2(I2020T) is denoted as \#\# for $P<0.001$ and \#\#\# for $P<0.0001$.

components of the retromer complex is specific to this particular mutation. To answer this question, we employed two other transgenic mutant LRRK2 lines: the LRRK2 (Y1699C) line carrying a confirmed PD pathogenic mutation in the COR domain of LRRK2 [26-28], and the $L R R K 2(I 1122 \mathrm{~V})$ line with a putative pathogenic mutation in the LRR domain [26,27].

Similar to LRRK2(I2020T), expressing either one of the two mutant forms of $L R R K 2$ in DA neurons caused a significant impairment locomotor activity on day 5 $(24.71 \%+/-3.44 \%$ of $\operatorname{LRRK2}(Y 1699 C)$ flies were able to reach the line within 5 seconds, compared to $63.21 \%+/$ $5.30 \%$ of control, $\mathrm{P}<0.0001 ; \mathrm{F}(3,177)=50.60$; and
$39.93 \%+/-2.73 \%$ of $\operatorname{LRRK2}(11122 \mathrm{~V})$ flies were able to reach the line within 5 seconds, compared to $72.75 \%+/$ $4.27 \%$ of control flies, $\mathrm{P}<0.0001$; $\mathrm{F}(3,126)=23.82$, respectively) (Figure 4A and B), which is consistent with our previous results [13]. Importantly, overexpressing Vps 35 in either one of the two LRRK2 mutant lines resulted in a complete rescue of the locomotor impairment $(74.40 \%+/-3.42 \%$ and $65.01 \%+/-3.55 \%$, respectively, of the double transgenic flies were able to reach the top within 5 seconds on day $5 ; \mathrm{P}<0.0001$ ) (Figure $4 \mathrm{~A}$ and $\mathrm{B}$ ). The results were very similar on Day 10 (Figure 4A and $\mathrm{B})$. These results demonstrate that the functional interaction between LRRK2 and Vps35 is not exclusive

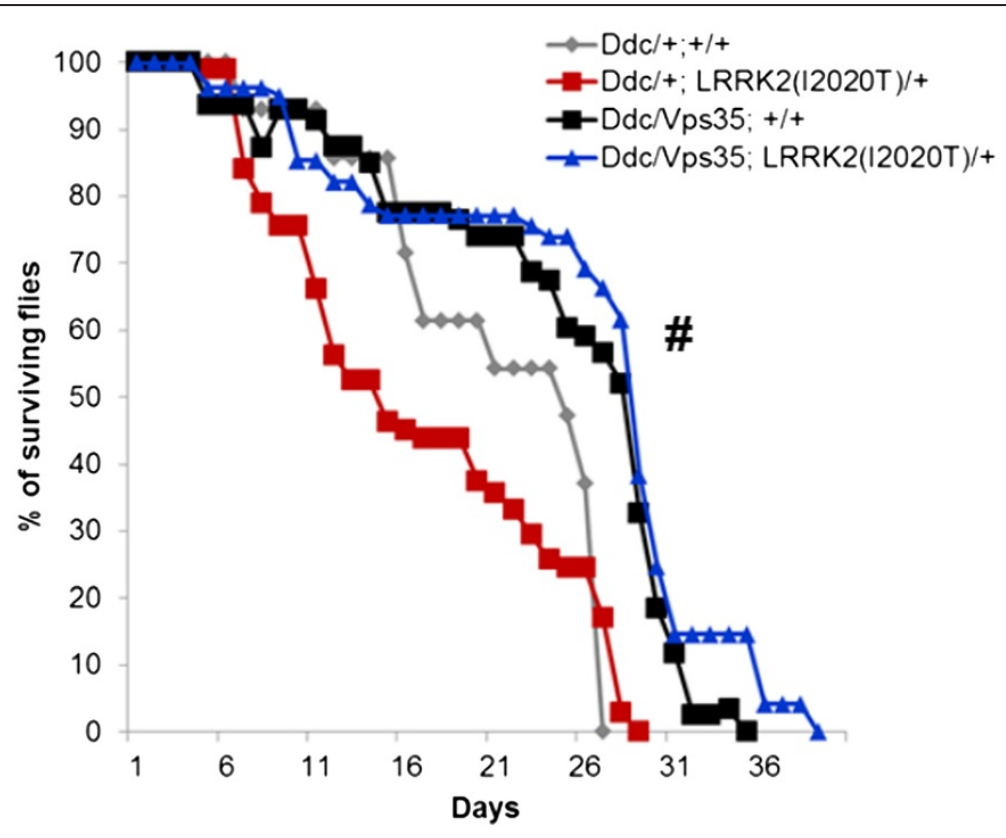

Figure 3 Overexpression of Vps35 in DA neurons rescues the shortened lifespan of LRRK2(I2020T) flies. N=8-11 cohorts of ten per genotype. All flies were reared at $29^{\circ} \mathrm{C}$. Statistical analysis: Two-Way ANOVA, Tukey's post-test. Statistically significant difference compared to Ddc/+,LRRK2(I2020T)/+is denoted as \# for $\mathrm{P}<0.05$. 
A

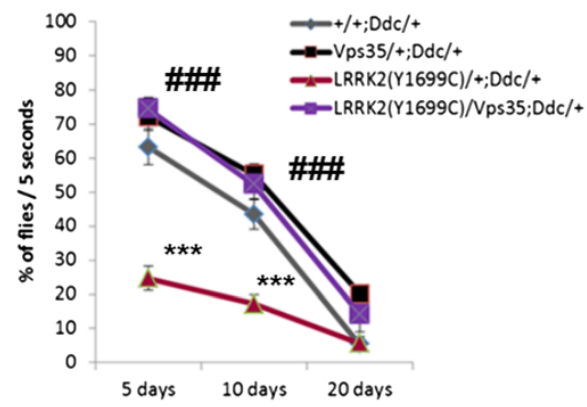

B

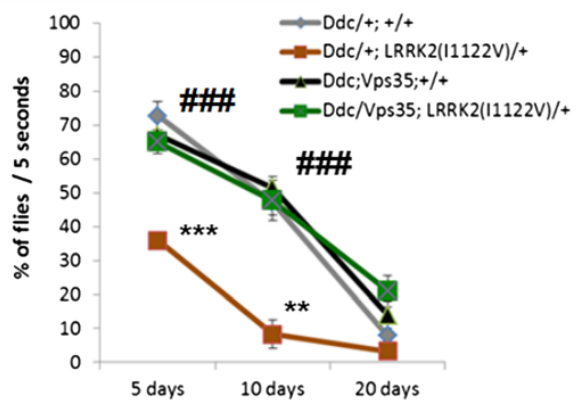

Figure 4 Overexpression of Vps35 in DA neurons rescues locomotor deficits of other PD mutants. (A) Overexpression of Vps35 in LRRK2 (Y1699C) mutants. (B) Overexpression of Vps35 in LRRK2(11122V) mutants. All flies were reared at $29^{\circ} \mathrm{C} . \mathrm{N}=3-7$ cohorts of ten. Statistical analysis: Two-Way ANOVA, Tukey's post-test. Statistically significant difference compared to control (Ddc alone) is denoted as ** for $\mathrm{P}<0.001$, or *** for $\mathrm{P}<0.0001$. Statistically significant difference compared to Ddc/t;LRRK2(I2020T)/+is denoted as \#\#\# for $\mathrm{P}<0.0001$.

to the kinase domain mutant, and provide further evidence that LRRK2 may play a role in the retromerdependent pathway.

\section{Knocking down components of the cargo-recognition} subunit of the retromer complex impairs locomotor activity Vps35 is the most recently confirmed causative gene of PD. However the mechanism by which mutation in Vps35 leads to PD is completely unknown. To better understand the importance of retromer for the physiological function of DA neurons, we analyzed the effect of knocking down expression of genes encoding for components of the retromer complex.

First, we analyzed the effect in the eye. Knocking down expression of Vps26 in the eye caused a significant eye phenotype $(35.84 \%+/-5.43 \%$ of Vps 26 knock-down eyes had a black lesion, compared to $2.78 \%+/-3.4 \%$ of control eyes; $\mathrm{P}<0.05$; $\mathrm{F}(3,15)=7.01)$ (Figure $5 \mathrm{~A}$ and $\mathrm{B}$ ). This phenotype was similar to the eye phenotype of the $L R R K 2$ (I2020T) mutant (44.71 +/- 6.49\% of LRRK2(I2020T) eyes had black lesions) (Figure 5A and B). Our next goal was to assess whether these two phenotypes are additive. An additive effect would indicate that the two genes likely act on two independent cellular pathways. We observed that the eye phenotypes of LRRK2(I2020T) expression and Vps26 knock-down were not additive $(36.21 \%+/$ - 3.02\% of double transgenic eyes had a black lesion) (Figure 5A and $\mathrm{B}$ ), suggesting that the two genes act on the same pathway.

The next step was to assess the effect of knocking down components of the retromer complex in DA neurons. In DA neurons, knocking-down expression of $V p s 35$ or Vps29 caused a significant impairment in the locomotor activity $(29.65 \%+/-2.44 \%$ and $40.68+/-2.93 \%$ of flies, respectively, reached the top within 5 seconds on day 5, compared to $72.75 \%+/-4.27 \%$ of control flies; $\mathrm{P}<0.0001, \mathrm{~F}(5,320)=9.335)$ (Figure $5 \mathrm{C})$. Similar to the effects seen in the eye, these climbing deficits were not additive with those observed in the LRRK2 (I2020T) mutants (Figure 5C), supporting our results that Vps35, Vps26 and Vps29 share a common pathway with $L R R K 2$.

\section{Vps35 protects from rotenone toxicity}

Rotenone is a pesticide and a complex I inhibitor that can be used to model parkinsonism [29]. In our experiments, we exposed the flies to rotenone to assess the role of LRRK2-Vps35 interaction under conditions of additional cellular stress.

Treating control flies with rotenone has a profound effect on their survival. Although we previously showed that pan-neuronal expression of LRRK2(I2020T) sensitized flies to low doses of rotenone [13], overexpression of LRRK2(I2020T) in DA neurons did not seem to significantly affect survival of flies treated with $1 \mathrm{mM}$ rotenone (Figure 6A). Importantly, overexpressing Vps35 alone offered a mild but significant protection against rotenone, compared to control flies (Figure 6A). To our surprise however, co-overexpressing Vps35 and LRRK2 in DA neurons resulted in a substantial synergistic protective effect against rotenone, demonstrated as a significantly better survival of these flies compared to all other groups $(\mathrm{P}<0.0001 ; \mathrm{F}(74,190)=3.45)$ (Figure 6A).

Next, we assessed the climbing ability of the rotenonetreated flies. Rotenone-treated LRRK2(I2020T) flies had somewhat unexpectedly a slightly higher locomotor activity compared to control flies (Figure 6B). Again, Vps35 overexpression alone significantly improved locomotor activity of the rotenone-treated flies (Figure 6B) and this effect was sustained even in the presence of LRRK2 $(I 2020 T)(\mathrm{P}=0.0011 ; \mathrm{F}(30,271)=2.10)($ Figure 6B).

Because Vps35 overexpression protected from rotenone, our next goal was to test whether silencing Vps35 would sensitize the flies to rotenone toxicity. This would suggest 


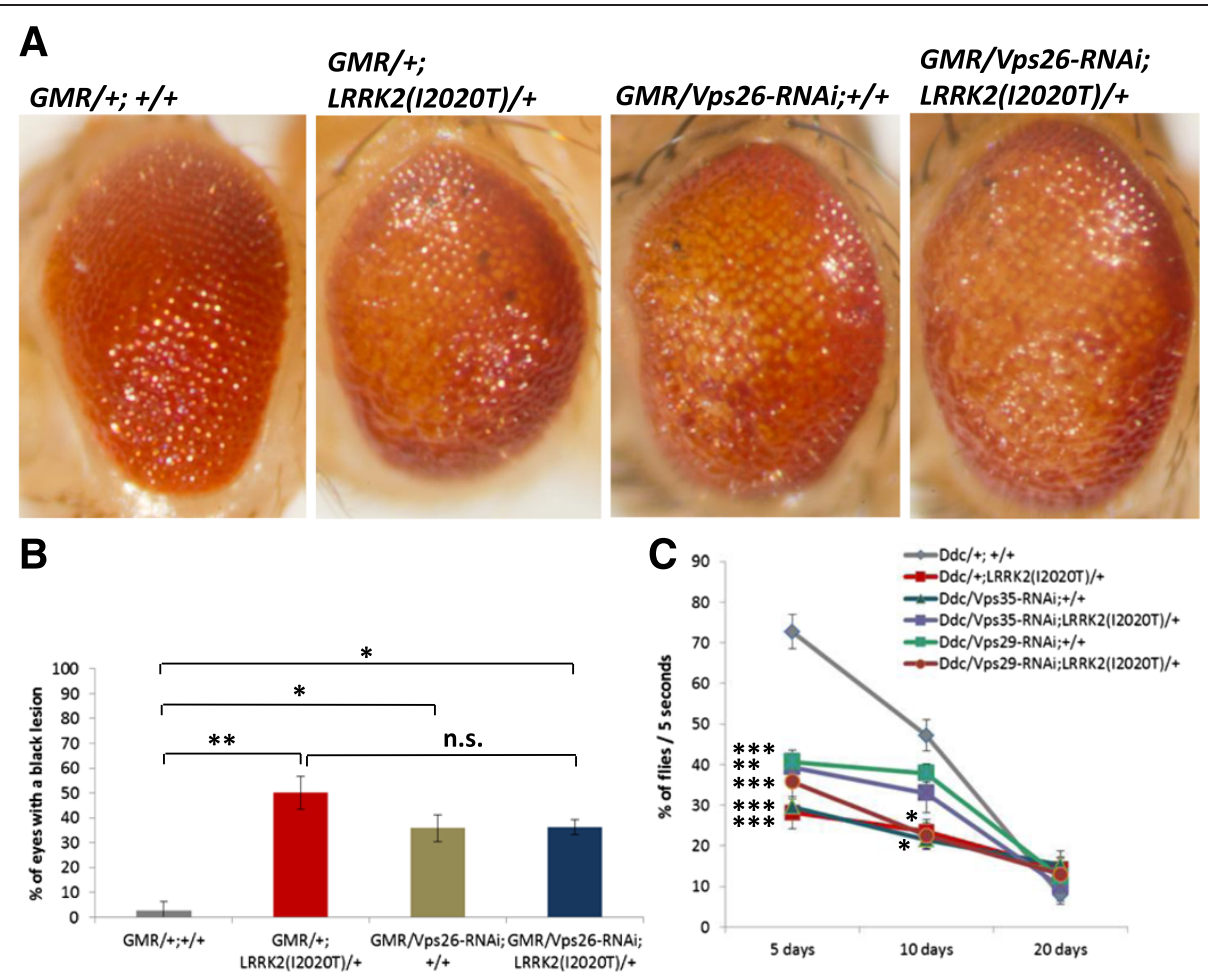

Figure 5 Knocking down components of the cargo-recognition subunit of the retromer complex causes an eye phenotype and a locomotor impairment. The phenotypes are not additive with LRRK2(I2020T) phenotypes. A) Representative stereomicroscope images and B) Quantification of black lesions in the eyes Vps26 knock-down flies. Total of 444 eyes from 3-9 independent crosses/genotype (males and females) was analyzed. Statistically significant difference compared to control (GMR alone) is denoted as * for $P<0.05$ and ** for $P<0.001$. Data were statistically analyzed by One-Way ANOVA, Bonferroni's post-test. C) Effect of Vps35 or Vps29 knock-down on locomotor activity. N=3-13 cohorts of ten. Statistical analysis: Two-Way ANOVA, Tukey's post-test. Statistically significant difference compared to control (Ddc alone) is denoted as ${ }^{*}$ for $P<0.05$, ** for $P<0.001$, or ${ }^{* *}$ for $P<0.0001$. All flies were reared at $29^{\circ} \mathrm{C}$.

that endogenous Vps35 is involved in the cellular protection against rotenone. However, our results show that knocking-down Vps35 had no statistically significant effect on climbing or survival of rotenone-treated flies, compared to control (Figure 6A and $\mathrm{C}$ ).

Altogether, these data point to a strong functional interaction between $L R R K 2$ and Vps35 in DA neurons which may be especially important under conditions of cellular stress. Furthermore, our data for the first time suggest that rotenone interferes with the endolysosomal pathway, although the exact mechanism is not clear.

In summary, we present evidence that overexpression of Drosophila Vps35, a core component of the retromer complex, rescues the eye phenotype, locomotor deficits and shortened lifespan of the LRRK2(I2020T) expressing flies. Similar to Vps35, overexpressing Vps26 rescued the eye and locomotor phenotypes, thus validating our findings. Moreover, we confirmed that overexpression of Vps35 also rescues the locomotor phenotypes of two other LRRK2 mutants. Furthermore, we demonstrate that knocking down Vps35 leads to a similar degree of locomotor impairment and eye damage as the mutant $L R R K 2$, but that the phenotypes of the two genes are not additive.
Finally, we show that while silencing $V p s 35$ has no significant effect on locomotor activity or survival of rotenonetreated flies, overexpressing Vps35 alone protects from cellular stress caused by rotenone, as demonstrated by prolonging lifespan and improving locomotor deficits, and that this protection by Vps35 is sustained and even augmented in the presence of mutant LRRK 2 .

\section{Discussion}

Several previous studies have indicated that LRRK2 plays a role in the endolysosomal trafficking [30,31], protein sorting and transport [32] or trafficking of synaptic vesicles [31,33]. For example, overexpression of $L R R K 2$ is associated with enlarged lysosomes, vacuolization and/or large cytoplasmic punctate structures [30,34,35], suggesting a problem with vesicular trafficking. Here we present evidence that LRRK2 and Vps35 functionally interact, and demonstrate how this interaction in DA neurons affects locomotor activity, lifespan and response to rotenone.

$L R R K 2$ is the most common cause of the monogenic form of PD, and a common risk factor for PD. To further highlight the relevance of our data to $\mathrm{PD}$, the human homologue of Vps35, VPS35, has recently been identified 


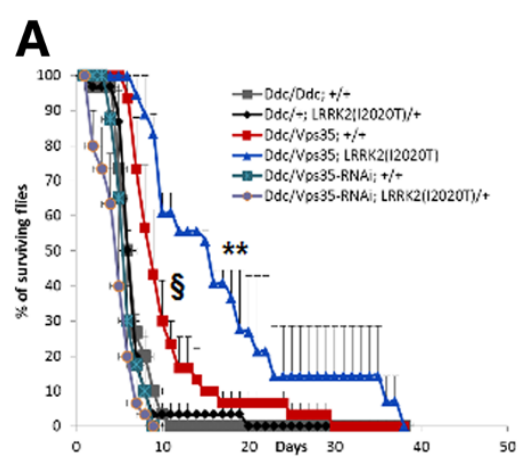

\section{B}
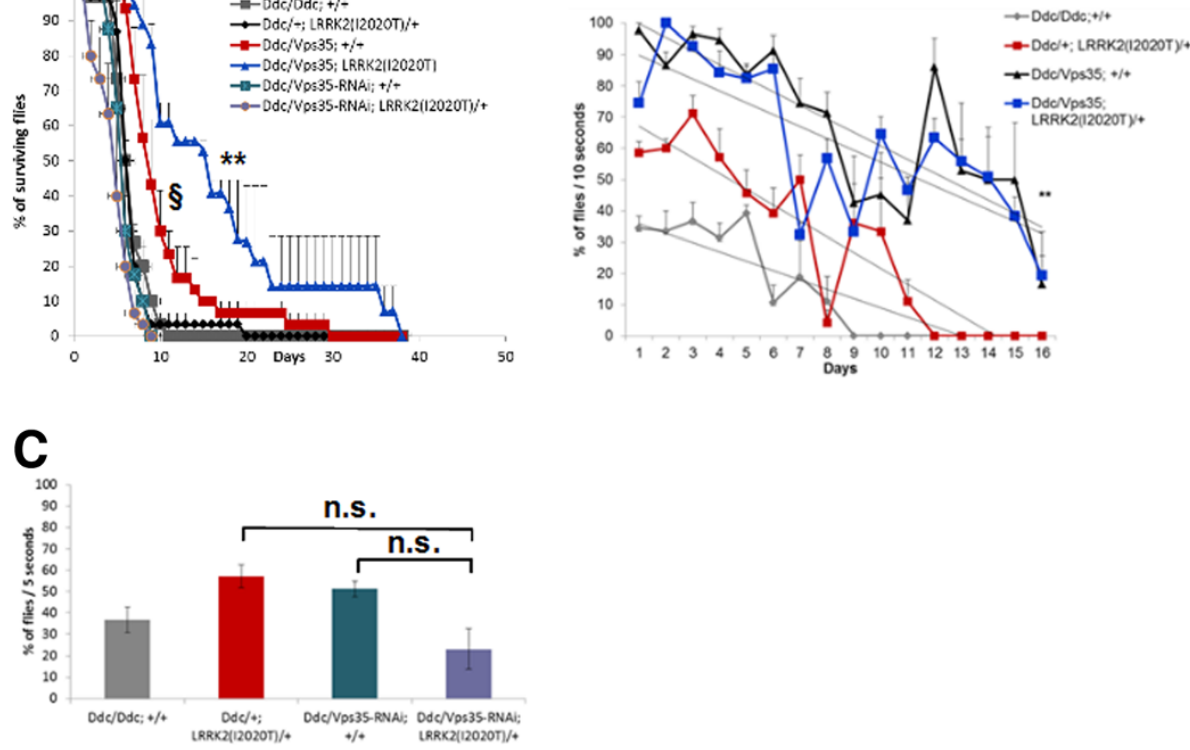

Figure 6 Effect of Vps35 overexpression and Vps35 knockdown on rotenone toxicity. A) Vps35 overexpression in DA neurons improves survival rates following exposure to rotenone, and LRRK2(I2020T) and Vps35 act synergistically to protect from rotenone. B) Vps35 overexpression in DA neurons protects from locomotor deficits seen in LRRK2(I2020T) or control flies exposed to rotenone. Knocking down Vps35 has no significant effect on survival (A), or locomotor activity (C). N=20-40 flies/genotype. Statistical analysis by Two-Way ANOVA, Tukey's post-test. Statistically significant difference compared to Ddc/t;LRRK2(I2020T) and control (Ddc alone) was denoted as * *for $\mathrm{P}<0.001$; statistically significant difference compared to control and compared to Ddc/Vps35;LRRK2(I2020T)/+was denoted as $\S$ for $P<0.05$.

as the latest confirmed causative gene of the typical late onset PD, with c.1858 > A (p.Asp620Asn) being the most common VPS35 mutation [36,37]. This finding has been replicated by several independent analyses [38-42]. However, the mechanism by which VPS35 is involved in PD pathogenesis is entirely unknown.

Our data indicate that $L R R K 2$ and components of the cargo-recognition subunits of the retromer complex Vps35 are part of the same molecular pathway, with mutant LRRK2 likely being upstream and negatively regulating retromer. Furthermore, our gene knock-down data suggest that the mechanism by which the PD pathogenic mutant VPS35 is involved in PD pathogenesis may be a dominant negative mechanism.

VPS35 is a core component of the evolutionarily conserved retromer complex that is predominantly expressed on dynamic endosomal membranes [43-45], to regulate sorting, packaging and directing transport of specific proteins to the trans-Golgi network or cell surface. Thus, the retromer complex prevents specific proteins from being degraded in the lysosome [46]. Retromer consists of two subcomplexes: a cargo recognition subcomplex composed of VPS35, VPS26 and VPS29 [47], and a membraneinteracting subcomplex composed of sorting nexins (SNXs) that bind to a PI3-P-rich endosomal membrane [48]. By regulating protein sorting, retromer is involved in many diverse cellular processes, including but not limited to trafficking of SNARE proteins and receptors such as $\beta$-adrenergic [49] or cation-independent mannose 6-phosphate receptors [50], or regulating homeostasis of intracellular glucose, copper and iron.

Although its physiological function in neuronal cells is not yet fully elucidated, it is clear that the retromerdependent pathway plays a role in etiology or pathophysiology of a number of neurodegenerative processes. For example, genetic variations of SorLa [51] or sorCS1 [52], encoding for receptors that are cargoes for the retromerdependent pathway, are associated with Alzheimer's disease, expression of VPS35, VPS26, sortilin and SorLa is altered in Alzheimer's disease patients [53,54] and interfering with expression of VPS26 or VPS35 [55] causes accumulation of amyloid $\beta$ and APP derivatives in exosomal compartments [56]. Further research into this pathway may therefore offer important clues and insights into the pathogenesis of PD and other neurodegenerative disorders.

MacLeod et al. recently published a paper that demonstrates an interaction between Vps 35 and another LRRK2 mutant, LRRK2(G2019S), where Vps35 protected against neuronal death caused by $L R R K 2(G 2019 S)$ [57]. Our data presented here independently validate these findings, 
and extend the relevance of these findings to two other pathogenic LRRK2 mutants, LRRK2(I2020T) and LRRK2 (Y1699C), and one putative pathogenic mutant LRRK2 $(I 1122 \mathrm{~V})$. More importantly however, we for the first time characterize the functional role of this interaction in regulation of the locomotor activity, lifespan, sensitivity to rotenone and in retinal degeneration. Furthermore, our data provide evidence that overexpressing Vps35 alone protects from rotenone, while knocking down Vps35 has no significant effect on rotenone toxicity. This is consistent with recently published data showing that overexpression of Vps35 protected in vitro against MPP+, another neurotoxin commonly used to model PD, but Vps35 knockdown had no significant effect on cell viability under the same conditions [58].

\section{Conclusions}

In summary, these data provide further evidence in support of the hypothesis that LRRK2 plays a role in the endolysosomal pathway and that the pathology caused by mutant LRRK2 may be at least partly linked to a disruption of this important protein sorting and recycling cellular process. However, how exactly this pathway contributes to PD pathology is at present entirely unknown. Elucidation of new molecular pathways involved in the pathogenesis of PD may bring forward novel pharmacological targets for better treatment strategies. Therefore, a better understanding of the retromer pathway and its relation to PD pathogenesis deserves further investigations.

\section{Materials and methods Drosophila genetics}

UAS-LRRK2(I2020T), UAS-LRRK2(Y1699C) and UASLRRK2(I1122V) lines were characterized previously [13]. $P$ $\{E P g y 2\} V p s 35^{E Y 14200} / C y O$ and P\{EP\}Vps26G2008 w*/FM7h flies were obtained from the Bloomington Drosophila Stock Center (BDSC, Indiana University). These lines contain an empty UAS element upstream of endogenous Drosophila Vps35 or Vps26 gene, respectively, allowing for a Gal4dependent cell/tissue specific gene overexpression $[59,60]$. Ddc-Gal4 and GMR-Gal4 lines were both obtained from BDSC. For the gene knockdown studies, we used the following lines from BDSC: $y 1 s c^{*} v 1$; P\{TRiP.HMS01858\} attP40 (expresses dsRNA for RNAi of Vps35 under $U A S$ control); y1 sc* v1; P\{TRiP.HMS01877\}attP40 (expresses dsRNA for RNAi of CG4764 (FBgn0031310) under UAS control), and $y 1$ v1; P\{TRiP.HMS01769\}attP40 (expresses dsRNA for RNAi of Vps26 under UAS control). To increase transgene expression under the temperaturesensitive UAS-Gal4 expression system [13], all flies were cultured on a standard cornmeal medium at $29^{\circ} \mathrm{C}(12-\mathrm{hrs}$ dark/light cycle), except for the rotenone-treated flies (see below).

\section{Eye phenotype}

To assess the eye phenotype, we used GMR-Gal4 to drive the expression of the transgenes in the eye. All crosses and $\mathrm{F} 1$ generations were reared at $29^{\circ} \mathrm{C}$. At 10 days of age, their eyes were analyzed under a stereomicroscope (Zeiss).

\section{Negative geotaxis assay and survival assay}

The transgenes were overexpressed in DA neurons under a $D d c$ promoter. Progeny of the appropriate genotype were divided into cohorts of ten, and the flies were subjected to a negative geotaxis climbing assay at 5, 10 and 20 days post-eclosure. We recorded and counted flies that crossed a line $8 \mathrm{~cm}$ above the base of a transparent tube within 5, 10 and 30 seconds after being gently tapped down. All behavioral experiments were performed at room temperature under standard light conditions. To ensure comparable conditions in each vial, we placed flies in vials with new food every 3-4 days. The same cohorts of flies used in the climbing assay were daily analyzed for survival.

\section{Rotenone treatment}

Cohorts of ten flies (five males and five females) were placed in vials containing freshly prepared rehydrated lyophilized food (Carolina Biological Supplies) containing rotenone ( $1 \mathrm{mM}$; Enzo, Farmingdale, NY). Flies were reared at room temperature, their survival and locomotor activity assessed daily, as described above. Every third day, the flies were transferred into a new vial with freshly prepared rotenone-containing food. Because rotenone is light- and temperature-sensitive, the flies were reared at room temperature and in the dark.

\section{Statistical analyses}

All data were analyzed by One-Way ANOVA with a Tukey's post-hoc test, or by Two-way ANOVA followed by a Bonferoni's post-hoc test, as indicated.

\section{Abbreviations \\ PD: Parkinson's disease; LRRK2: Leucine-rich repeat kinase 2; DA: Dopaminergic Vps35: Vacuolar protein sorting 35; Vps26: Vacuolar protein sorting 26; Vps29: Vacuolar protein sorting 29; BDSC: Bloomington Drosophila Stock Center; Ddc: Dopa-decarboxylase.}

\section{Competing interests}

The authors declare that they have no competing interests.

\section{Authors' contributions}

SAW, JC, MT, AH, CA, JMP, CH, ST, SS, RP and JK participated in carrying out the experiments, RL participated in the design of the experiments, in carrying out the experiments, data analysis and preparation of the manuscript, KV conceived, designed and coordinated the study, participated in carrying out the experiments, analyzed the data and drafted the manuscript. All authors read and approved the final manuscript. 


\section{Acknowledgements}

This work was partially funded by the Pacific Fund Summer Research Fellowship (SAW), and by the University of the Pacific SAAG and Eberhardt awards (KV). We wish to thank Dilraj Sohal, Erika Cho, Judy Ho, Emily Hou, Bryant Pham, Ariel Tsang, Brian Phi, Gina Stassinos, Edwin Yadidi, Ryan Fedrizzi, Derek Fong, Victoria Nguyen, Ravneet Kaur, Sukhjit Sagoo and Christine Choi from the University of the Pacific for their technical assistance with the experiments.

\section{Received: 9 September 2013 Accepted: 9 May 2014}

Published: 11 June 2014

\section{References}

1. Melrose HL, Dachsel JC, Behrouz B, Lincoln SJ, Yue M, Hinkle KM, Kent CB, Korvatska E, Taylor JP, Witten L, Liang YQ, Beevers JE, Boules M, Dugger BN, Serna VA, Gaukhman A, Yu X, Castanedes-Casey M, Braithwaite AT, Ogholikhan S, Yu N, Bass D, Tyndall G, Schellenberg GD, Dickson DW, Janus C, Farrer MJ Impaired dopaminergic neurotransmission and microtubule-associated protein tau alterations in human LRRK2 transgenic mice. Neurobiol Dis 2010, 40:503-517.

2. Carballo-Carbajal I, Weber-Endress S, Rovelli G, Chan D, Wolozin B, Klein CL, Patenge N, Gasser T, Kahle PJ: Leucine-rich repeat kinase 2 induces alpha-synuclein expression via the extracellular signal-regulated kinase pathway. Cell Signal 2010, 22:821-827.

3. Tong Y, Yamaguchi H, Giaime E, Boyle S, Kopan R, Kelleher RJ 3rd, Shen J: Loss of leucine-rich repeat kinase 2 causes impairment of protein degradation pathways, accumulation of alpha-synuclein, and apoptotic cell death in aged mice. Proc Natl Acad Sci U S A 2010, 107:9879-9884.

4. Lin X, Parisiadou L, Gu XL, Wang L, Shim H, Sun L, Xie C, Long CX, Yang WJ, Ding J, Chen ZZ, Gallant PE, Tao-Cheng JH, Rudow G, Troncoso JC, Liu Z, Li $\mathrm{Z}$, Cai H: Leucine-rich repeat kinase 2 regulates the progression of neuropathology induced by Parkinson's-disease-related mutant alpha-synuclein. Neuron 2009, 64:807-827.

5. Cookson MR, Bandmann O: Parkinson's disease: insights from pathways. Hum Mol Genet 2010, 19:R21-R27.

6. Feany MB, Bender WW: A drosophila model of Parkinson's disease. Nature 2000, 404:394-398.

7. Guo M: What have we learned from Drosophila models of Parkinson's disease? Prog Brain Res 2010, 184:3-16.

8. Pienaar IS, Gotz J, Feany MB: Parkinson's disease: insights from nontraditional model organisms. Prog Neurobiol 2010, 92:558-571.

9. Bilen J, Bonini NM: Genome-wide screen for modifiers of ataxin-3 neurodegeneration in Drosophila. PLoS Genet 2007, 3:1950-1964.

10. Karsten SL, Sang TK, Gehman LT, Chatterjee S, Liu J, Lawless GM, Sengupta S, Berry RW, Pomakian J, Oh HS, Schulz C, Hui KS, Wiedau-Pazos M, Vinters $H V$, Binder LI, Geschwind DH, Jackson GR: A genomic screen for modifiers of tauopathy identifies puromycin-sensitive aminopeptidase as an inhibitor of tau-induced neurodegeneration. Neuron 2006, 51:549-560.

11. Fernandes C, Rao Y: Genome-wide screen for modifiers of Parkinson's disease genes in Drosophila. Mol Brain 2011, 4:17.

12. Ambegaokar SS, Roy B, Jackson GR: Neurodegenerative models in Drosophila: polyglutamine disorders, Parkinson disease, and amyotrophic lateral sclerosis. Neurobiol Dis 2010, 40:29-39.

13. Venderova K, Kabbach G, Abdel-Messih E, Zhang Y, Parks RJ, Imai Y, Gehrke S, Ngsee J, Lavoie MJ, Slack RS, Rao Y, Zhang Z, Lu B, Haque ME, Park DS: Leucine-Rich Repeat Kinase 2 interacts with Parkin, DJ-1 and PINK-1 in a Drosophila melanogaster model of Parkinson's disease. Hum Mol Genet 2009, 18:4390-4404.

14. Kanao T, Venderova K, Park DS, Unterman T, Lu B, Imai Y: Activation of FoxO by LRRK2 induces expression of proapoptotic proteins and alters survival of postmitotic dopaminergic neuron in Drosophila. Hum Mol Genet 2010, 19:3747-3758.

15. Hindle S, Afsari F, Stark M, Middleton CA, Evans GJ, Sweeney ST, Elliott CJ: Dopaminergic expression of the Parkinsonian gene LRRK2-G2019S leads to non-autonomous visual neurodegeneration, accelerated by increased neural demands for energy. Hum Mol Genet 2013, 22:2129-2140.

16. Liu Z, Wang X, Yu Y, Li X, Wang T, Jiang H, Ren Q, Jiao Y, Sawa A, Moran T, Ross CA, Montell C, Smith WW: A Drosophila model for LRRK2-linked parkinsonism. Proc Natl Acad Sci U S A 2008, 105:2693-2698.
17. Smith WW, Pei Z, Jiang H, Moore DJ, Liang Y, West AB, Dawson VL, Dawson TM, Ross CA: Leucine-rich repeat kinase 2 (LRRK2) interacts with parkin, and mutant LRRK2 induces neuronal degeneration. Proc Natl Acad Sci U S A 2005, 102:18676-18681.

18. Ng CH, Mok SZ, Koh C, Ouyang X, Fivaz ML, Tan EK, Dawson VL, Dawson TM, Yu F, Lim KL: Parkin protects against LRRK2 G2019S mutant-induced dopaminergic neurodegeneration in Drosophila. J Neurosci 2009, 29:11257-11262

19. Brand AH, Perrimon N: Targeted gene expression as a means of altering cell fates and generating dominant phenotypes. Development 1993, 118:401-415.

20. Doumanis J, Wada K, Kino Y, Moore AW, Nukina N: RNAi screening in Drosophila cells identifies new modifiers of mutant huntingtin aggregation. PLoS One 2009, 4:e7275.

21. Korey CA, MacDonald ME: An over-expression system for characterizing Ppt1 function in Drosophila. BMC Neurosci 2003, 4:30.

22. Ahmad ST, Sweeney ST, Lee JA, Sweeney NT, Gao FB: Genetic screen identifies serpin 5 as a regulator of the toll pathway and CHMP2B toxicity associated with frontotemporal dementia. Proc Natl Acad Sci U S A 2009, 106:12168-12173.

23. Wang D, Qian L, Xiong H, Liu J, Neckameyer WS, Oldham S, Xia K, Wang J, Bodmer R, Zhang Z: Antioxidants protect PINK1-dependent dopaminergic neurons in Drosophila. Proc Natl Acad Sci U S A 2006, 103:13520-13525.

24. Li Y, Ray P, Rao EJ, Shi C, Guo W, Chen X, Woodruff EA 3rd, Fushimi K, Wu JY: A Drosophila model for TDP-43 proteinopathy. Proc Natl Acad SCi U S A 2010, 107:3169-3174

25. Liu Z, Hamamichi S, Lee BD, Yang D, Ray A, Caldwell GA, Caldwell KA, Dawson TM, Smith WW, Dawson VL: Inhibitors of LRRK2 kinase attenuate neurodegeneration and Parkinson-like phenotypes in Caenorhabditis elegans and Drosophila Parkinson's disease models. Hum Mol Genet 2011, 20:3933-3942.

26. Paisan-Ruiz C, Jain S, Evans EW, Gilks WP, Simon J, van der Brug M, Lopez de Munain A, Aparicio S, Gil AM, Khan N, Johnson J, Martinez JR, Nicholl D, Carrera IM, Pena AS, de Silva R, Lees A, Marti-Masso JF, Perez-Tur J, Wood NW, Singleton AB: Cloning of the gene containing mutations that cause PARK8-linked Parkinson's disease. Neuron 2004, 44:595-600.

27. Zimprich A, Biskup S, Leitner $P$, Lichtner P, Farrer M, Lincoln S, Kachergus J, Hulihan M, Uitti RJ, Calne DB, Stoessl AJ, Pfeiffer RF, Patenge N, Carbajal IC, Vieregge P, Asmus F, Muller-Myhsok B, Dickson DW, Meitinger T, Strom TM, Wszolek ZK, Gasser T: Mutations in LRRK2 cause autosomal-dominant parkinsonism with pleomorphic pathology. Neuron 2004, 44:601-607.

28. Singleton $A B$, Farrer MJ, Bonifati V: The genetics of Parkinson's disease: progress and therapeutic implications. Mov Disord 2013, 28:14-23.

29. Jackson-Lewis V, Blesa J, Przedborski S: Animal models of Parkinson's disease. Parkinsonism Relat Disord 2012, 18(Suppl 1):S183-S185.

30. Xiong Y, Coombes CE, Kilaru A, Li X, Gitler AD, Bowers WJ, Dawson VL, Dawson TM, Moore DJ: GTPase activity plays a key role in the pathobiology of LRRK2. PLoS Genet 2010, 6:e1000902.

31. Piccoli G, Condliffe SB, Bauer M, Giesert F, Boldt K, De Astis S, Meixner A, Sarioglu H, Vogt-Weisenhorn DM, Wurst W, Gloeckner CJ, Matteoli M, Sala C, Ueffing M: LRRK2 controls synaptic vesicle storage and mobilization within the recycling pool. J Neurosci 2011, 31:2225-2237.

32. Sakaguchi-Nakashima A, Meir JY, Jin Y, Matsumoto K, Hisamoto N: LRK-1, a C. elegans PARK8-related kinase, regulates axonal-dendritic polarity of SV proteins. Curr Biol 2007, 17:592-598.

33. Shin $\mathrm{N}$, Jeong H, Kwon J, Heo HY, Kwon JJ, Yun HJ, Kim CH, Han BS, Tong Y, Shen J, Hatano T, Hattori N, Kim KS, Chang S, Seol W: LRRK2 regulates synaptic vesicle endocytosis. Exp Cell Res 2008, 314:2055-2065.

34. MacLeod D, Dowman J, Hammond R, Leete T, Inoue K, Abeliovich A: The familial Parkinsonism gene LRRK2 regulates neurite process morphology. Neuron 2006, 52:587-593.

35. Plowey ED, Cherra SJ 3rd, Liu YJ, Chu CT: Role of autophagy in G2019S-LRRK2-associated neurite shortening in differentiated SH-SY5Y cells. J Neurochem 2008, 105:1048-1056.

36. Vilarino-Guell C, Wider C, Ross OA, Dachsel JC, Kachergus JM, Lincoln SJ, Soto-Ortolaza Al, Cobb SA, Wilhoite GJ, Bacon JA, Behrouz B, Melrose HL, Hentati E, Puschmann A, Evans DM, Conibear E, Wasserman WW, Aasly JO, Burkhard PR, Djaldetti R, Ghika J, Hentati F, Krygowska-Wajs A, Lynch T, Melamed E, Rajput A, Rajput AH, Solida A, Wu RM, Uitti RJ, et al: VPS35 mutations in Parkinson disease. Am J Hum Genet 2011, 89:162-167. 
37. Zimprich A, Benet-Pages A, Struhal W, Graf E, Eck SH, Offman MN, Haubenberger D, Spielberger S, Schulte EC, Lichtner P, Rossle SC, Klopp N, Wolf E, Seppi K, Pirker W, Presslauer S, Mollenhauer B, Katzenschlager R, Foki T, Hotzy C, Reinthaler E, Harutyunyan A, Kralovics R, Peters A, Zimprich F, Brucke T, Poewe W, Auff E, Trenkwalder C, Rost B, et al: A mutation in VPS35, encoding a subunit of the retromer complex, causes late-onset Parkinson disease. Am J Hum Genet 2011, 89:168-175.

38. Lesage S, Condroyer C, Klebe S, Honore A, Tison F, Brefel-Courbon C, Durr A, Brice A, French Parkinson's Disease Genetics Study G: Identification of VPS35 mutations replicated in French families with Parkinson disease. Neurology 2012, 78:1449-1450.

39. Kumar KR, Weissbach A, Heldmann M, Kasten M, Tunc S, Sue CM, Svetel M, Kostic VS, Segura-Aguilar J, Ramirez A, Simon DK, Vieregge P, Munte TF, Hagenah J, Klein C, Lohmann K: Frequency of the D620N mutation in VPS35 in Parkinson disease. Arch Neurol 2012, 69:1360-1364.

40. Ando M, Funayama M, Li Y, Kashihara K, Murakami Y, Ishizu N, Toyoda C, Noguchi K, Hashimoto T, Nakano N, Simon DK, Vieregge P, Munte TF, Hagenah J, Klein C, Lohmann K: VPS35 mutation in Japanese patients with typical Parkinson's disease. Mov Disord 2012, 27:1413-1417.

41. Sharma M, loannidis JP, Aasly JO, Annesi G, Brice A, Bertram L, Bozi M, Barcikowska M, Crosiers D, Clarke CE, Facheris MF, Farrer M, Garraux G, Gispert S, Auburger G, Vilarino-Guell C, Hadjigeorgiou GM, Hicks AA, Hattori N, Jeon BS, Jamrozik Z, Krygowska-Wajs A, Lesage S, Lill CM, Lin JJ, Lynch T, Lichtner $P$, Lang AE, Libioulle $C$, Murata $M$, et al: A multi-centre clinico-genetic analysis of the VPS35 gene in Parkinson disease indicates reduced penetrance for disease-associated variants. J Med Genet 2012, 49:721-726.

42. Nuytemans K, Bademci G, Inchausti V, Dressen A, Kinnamon DD, Mehta A, Wang L, Zuchner S, Beecham GW, Martin ER, Scott WK, Vance JM: Whole exome sequencing of rare variants in EIF4G1 and VPS35 in Parkinson disease. Neurology 2013, 80:982-989.

43. Arighi CN, Hartnell LM, Aguilar RC, Haft CR, Bonifacino JS: Role of the mammalian retromer in sorting of the cation-independent mannose 6-phosphate receptor. J Cell Biol 2004, 165:123-133.

44. Seaman MN: Cargo-selective endosomal sorting for retrieval to the Golgi requires retromer. J Cell Biol 2004, 165:111-122.

45. Haft CR, de la Luz SM, Bafford R, Lesniak MA, Barr VA, Taylor SI: Human orthologs of yeast vacuolar protein sorting proteins Vps26, 29, and 35 : assembly into multimeric complexes. Mol Biol Cell 2000, 11:4105-4116.

46. Lieu ZZ, Gleeson PA: Endosome-to-Golgi transport pathways in physiological processes. Histol Histopathol 2011, 26:395-408.

47. Seaman MN, McCaffery JM, Emr SD: A membrane coat complex essential for endosome-to-Golgi retrograde transport in yeast. J Cell Biol 1998 142:665-681.

48. Wassmer T, Attar N, Bujny MV, Oakley J, Traer CJ, Cullen PJ: A loss-of-function screen reveals SNX5 and SNX6 as potential components of the mammalian retromer. J Cell Sci 2007, 120:45-54.

49. Temkin $P$, Lauffer B, Jager S, Cimermancic $P$, Krogan NJ, von Zastrow M: SNX27 mediates retromer tubule entry and endosome-to-plasma membrane trafficking of signalling receptors. Nat Cell Biol 2011, 13:715-721.

50. Seaman MN: Identification of a novel conserved sorting motif required for retromer-mediated endosome-to-TGN retrieval. J Cell Sci 2007, 120:2378-2389.

51. Rogaeva E, Meng Y, Lee JH, Gu Y, Kawarai T, Zou F, Katayama T, Baldwin CT,

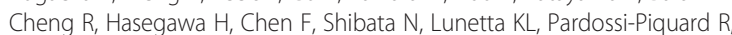
Bohm C, Wakutani Y, Cupples LA, Cuenco KT, Green RC, Pinessi L, Rainero I, Sorbi S, Bruni A, Duara R, Friedland RP, Inzelberg R, Hampe W, Bujo H, Song $Y Q$, Andersen OM, et al: The neuronal sortilin-related receptor SORL1 is genetically associated with Alzheimer disease. Nat Genet 2007, 39:168-177.

52. Liang X, Slifer M, Martin ER, Schnetz-Boutaud N, Bartlett J, Anderson B, Zuchner S, Gwirtsman H, Gilbert JR, Pericak-Vance MA, Haines JL: Genomic convergence to identify candidate genes for Alzheimer disease on chromosome 10. Hum Mutat 2009, 30:463-471.

53. Scherzer CR, Offe K, Gearing M, Rees HD, Fang G, Heilman CJ, Schaller C, Bujo H, Levey Al, Lah JJ: Loss of apolipoprotein E receptor LR11 in Alzheimer disease. Arch Neurol 2004, 61:1200-1205.

54. Finan GM, Okada H, Kim TW: BACE1 retrograde trafficking is uniquely regulated by the cytoplasmic domain of sortilin. J Biol Chem 2011, 286:12602-12616.
55. Muhammad A, Flores I, Zhang H, Yu R, Staniszewski A, Planel E, Herman M, Ho L, Kreber R, Honig LS, Ganetzky B, Duff K, Arancio O, Small SA: Retromer deficiency observed in Alzheimer's disease causes hippocampal dysfunction, neurodegeneration, and Abeta accumulation. Proc Natl Acad Sci U S A 2008, 105:7327-7332.

56. Sullivan CP, Jay AG, Stack EC, Pakaluk M, Wadlinger E, Fine RE, Wells JM, Morin PJ: Retromer disruption promotes amyloidogenic APP processing. Neurobiol Dis 2011, 43:338-345.

57. Macleod DA, Rhinn H, Kuwahara T, Zolin A, Di Paolo G, Maccabe BD, Marder KS, Honig LS, Clark LN, Small SA, Abeliovich A: RAB7L1 interacts with LRRK2 to modify intraneuronal protein sorting and Parkinson's disease risk. Neuron 2013, 77:425-439.

58. Bi F, Li F, Huang C, Zhou H: Pathogenic mutation in VPS35 impairs its protection against MPP(+) cytotoxicity. Int J Biol Sci 2013, 9:149-155.

59. Korolchuk VI, Schutz MM, Gomez-Llorente C, Rocha J, Lansu NR, Collins SM, Wairkar YP, Robinson IM, O'Kane CJ: Drosophila Vps35 function is necessary for normal endocytic trafficking and actin cytoskeleton organisation. J Cell Sci 2007, 120:4367-4376.

60. Bellen HJ, Levis RW, Liao G, He Y, Carlson JW, Tsang G, Evans-Holm M, Hiesinger PR, Schulze KL, Rubin GM, Hoskins RA, Spradling AC: The BDGP gene disruption project: single transposon insertions associated with 40\% of Drosophila genes. Genetics 2004, 167:761-781.

doi:10.1186/1750-1326-9-23

Cite this article as: Linhart et al:: Vacuolar protein sorting 35 (Vps35) rescues locomotor deficits and shortened lifespan in Drosophila expressing a Parkinson's disease mutant of Leucine-rich repeat kinase 2 (LRRK2). Molecular Neurodegeneration 2014 9:23.

\section{Submit your next manuscript to BioMed Central and take full advantage of:}

- Convenient online submission

- Thorough peer review

- No space constraints or color figure charges

- Immediate publication on acceptance

- Inclusion in PubMed, CAS, Scopus and Google Scholar

- Research which is freely available for redistribution 